\title{
PAPEL ACTUAL DEL ANTÍGENO PROSTÁtICO ESPECÍFICO Y OTROS MARCADORES EN EL DIAGNÓSTICO DEL CÁNCER DE PRÓSTATA.
}

\author{
F. Gómez Veiga, J. Ponce Reixa, A. Barbagelata López, E. Fernández Rosado y \\ M. González Martín.
}

Servicio de Urología. Complejo Hospitalario Universitario Juan Canalejo A Coruña. España.

\begin{abstract}
Resumen.- OBJETIVOS: Valorar el papel actual del PSA como método de diagnóstico en el cáncer de próstata y analizar los avances con nuevos marcadores relacionados con dicho tumor.

MÉTODOS: Revisamos en la literatura el valor del PSA como marcador para definir la presencia de cáncer de próstata, así como sus fórmulas moleculares. Las factores relacionados con sus modificaciones, modelos predictivos o las diferentes discrepancias en la utilidad sobre el nivel de corte para definir riesgo o como marcador en si mismo. Analizamos los posibles nuevos marcadores o líneas más interesantes de trabajo en el desarrollo de nuevos test. Utilizamos como vía de trabajo fundamental para la búsqueda bibliografica el Medline.
\end{abstract}

RESULTADOS: Los datos disponibles confirman que el PSA mantiene un nivel alto de sensibilidad aunque la especificidad es baja especialmente en rango de $P S A \leq 10 \mathrm{ngr} / \mathrm{ml}$, se puede ver aumentada con sus diferentes isoformas moleculares, ratios o modelos predictivos. Si bien es verdad que a pesar de dichos estudios persiste la dificultad para aumentar la especificidad y por lo tanto obviar biopsias. En la actualidad se dispone de nuevos marcadores, algunos comercializados y otros en vía de desarrollo como las nuevas isoformas o con biología molecular, que parecen mejorar la especificidad del PSA.

CONCLUSIONES: EI PSA sigue siendo el marcador patrón para el diagnóstico del cáncer de próstata. Es importante mejorar la especificidad para lo que necesitamos nuevos modelos predictivos o nuevas isoformas que puedan ayudarnos a seleccionar mejor los pacientes candidatos a biopsia. Existen en este momento diferentes líneas de investigación prometedoras con nuevos marcadores, si bien aún no existe sustituto ideal para el PSA que sigue siendo el patron estándar.

Palabras clave: PSA. Cáncer de Próstata.
F. Gómez Veiga Servicio de Urología Complejo Hospitalario Universitario Juan Canalejo Las Jubias 15009 A Coruña. (España) fgveiga@telefonica.net
Summary.- OBJECTIVES: To evaluate the current role of PSA as a diagnostic method for prostate cancer, as well as to analyze possible new markers.

METHODS: We perform a bibliographic review for PSA, and its molecular forms, as a marker to define the presence of prostate cancer. We review the factors related to PSA modifications, predictive models, or the current controversies about the usefulness of its cutpoint to define the risk of prostate cancer or the marker itself. 
We analyze possible new markers and the most interesting work lines in the development of new markers. We used MEDLINE for the bibliographic search.

RESULTS: Available data confirm that PSA has a high sensitivity; although specificity is low, mainly in the $\leq 10$ $\mathrm{ng} / \mathrm{m} /$ range, it may be increased with the use of various molecular isoforms, ratios or predictive models. Nevertheless, it is true that despite such studies it is difficult to increase specificity, so biopsies are reduced. Currently we have new markers, some of them already marketed, others in development, which seem to improve the specificity of PSA (isoforms, use of molecular biology).

CONCLUSIONS: PSA is still the standard marker for the diagnosis of prostate cancer. It is important to improve the specificity; therefore we need new predictive models or new isoforms that help us to do a better selection of candidates for biopsy. There are various promising research lines with new markers, but there is not ideal substitute for PSA yet.

Keywords: PSA. Prostatic cancer.

\section{INTRODUCCIÓN}

El descubrimiento del PSA se consolida con la purificación de la proteína de tejido prostático que se publica en 1979 (1), si bien este es el hallazgo clave, previamente un grupo japonés en 1971 (2) descubre la proteína en líquido seminal y su utilización se relaciona como posible elemento para diagnoóstico en casos de violación.

La FDA da su aprobación inicialmente para la monitorización para el cáncer de próstata en 1986. y no es hasta la década de los 90 en concreto en 1994 cuando lo aprueba para su diagnóstico (precoz o despistaje sistemático) (3). No se puede perder de vista en este análisis histórico de hechos la confluencia de factores que potencian la validad del PSA para la mejora del diagnóstico de cáncer de próstata. Uno de ellos es la incorporación de los equipos de ecografía y la introducción de la biopsia prostática ecodirigida como una rutina sistematizada que dará como resultado una mejoría clara en el diagnóstico (4). Todo ello incrementado con la evolución de la técnica quirúrgica y las técnicas de radioterapia externa o intersticial, darán como resultado que aproximadamente entre 1974 y 1993 el número de pacientes que fueron tratados por prostatectomía radical o alguna forma de radioterapia se triplicasen (3).

En la actualidad más de 200.000 nuevos casos se diagnostican por año en Europa y EEUU, siendo la primera neoplasia en el varón y en este momento la tercera causa de muerte en $\operatorname{EEUU}(5,6)$. En España fallecieron por dicha causa más de 5600 varones en 2004 (7). En cualquier caso la aplicación intensiva del PSA hace que hoy en día la mayoría de los diagnósticos de los tumores sean localizados y más del $60 \%$ de los tumores detectados están en estadios Tlc (8).

En esta revisión analizaremos la evolución del PSA así como las controversias generadas sobre el mismo junto con un análisis de las nuevas vías en el estudio de nuevos marcadores.

\section{BIOQUÍMICA}

El PSA es una glicoproteina perteneciente al grupo de las kalicreínas, producida básicamente por las células epiteliales situadas en los tejidos prostáticos y líquido seminal, alojadas en el cromososma 19 q 13.4 (9) . Aproximadamente entre un $70 \%$ y $90 \%$ del PSA se presenta de forma compleja (PSAc) ligado al $\alpha-1$ antiquimiotripsina y una menor proporción al $\alpha 1$ antitripsina (10). El PSA libre (PSAl) corresponde a un $10 \%$ un $30 \%$ del PSA y es una forma generalmente inactiva y esta a su vez puede presentar varias isoformas como se ha visto recientemente. El PSA en sangre periférica en gran parte está presente como forma inactiva. El PSA libre en gran medida se presenta como forma inactiva. Recientemente nuevas isoformas derivadas de este PSA libre han sido descubiertas, las conocidas como bPSA originario de la porción benigna de la próstata (zona transicional) y el proPSA o más relacionado con la presencia de tumor o zona periférica (11). En cualquier caso la porción del PSA en sus diferentes formas puede interponerse o entremezclarse tanto en tejido benigno como tumoral si bien alguna de las formas compleja, libre y recientemente estas nuevas isoformas de la porción inactiva pudieran ser más específicas para detectar cáncer. (Figura 1)

\section{PSA e ISOFORMAS "Libre y Complejada"}

EI PSA tras la aprobación por la FDA en 1986 para el seguimiento del cáncer de próstata y en particular para el despistaje sistemático en el 1994 ha demostrado la gran capacidad para detectar tumores. El trabajo de Stamey (12) publicado en 1987 es el referente que marca la era del PSA. Dicho trabajo relaciona claramente incremento del psa con la presencia de tumor así como su correlación con el volumen y el estadío.

Este estudio abrió las vías de nuevos trabajos en el desarrollo del PSA. En 1991 Catalona (13) 
pone de manifiesto la importancia del PSA para el diagnóstico precoz en combinación con tacto rectal y la ecografía. Con 1693 pacientes analizados se definen algunos datos importantes como el hecho de establecer "rangos". Así entre 4-10ng/ml de PSA, $22 \%$ de los pacientes presentaban tumor frente al $67 \%$ de los pacientes con PSA>10. Así mismo este estudio reseña lo que posteriormente será referencia en múltiples series y es el señalar la relación entre PSA y presencia o no de tumores organo-confinados, siendo para los pacientes con PSA entre $4-10 \mathrm{ng} / \mathrm{ml}$ la enfermedad organo-confinada en un $79 \%$ frente a un $13 \%$ de los que presentaban PSA $>10 \mathrm{ng} / \mathrm{ml}$.

El PSA en el inicio de la década de los 90 queda definido como marcador demostrando la conveniencia de usar un corte de 4 para definir pacientes candidatos a biopsia, así como establecer un rango de 4-10 denominado por la especificidad del PSA "zona gris" y PSA>10 se asocian con frecuentes porcentajes de tumores.

La relativa baja especificidad y el estudio de las fórmulas moleculares del PSA abren en la década de los 90 la vía de los que son hoy las más conocidas del PSA "PSAI y PSAc".

\section{PSA Libre}

Lija y su grupo en el 90 descubre las formas complejas y libres del PSA, asociando una disminución de proporción del PSAI en pacientes con patología benigna 10. Posteriores estudios detectan la mayor especificidad del PSAl y sus ratios sobre el PSA total (Tabla I) (15-21). El estudio multicéntrico publicado en 1998 con 773 pacientes confirman que el PSAl con el ratio de $25 \%$ detecta el $95 \%$ de los tumores ahorrando un $20 \%$ de biopsias. También se observa como el porcentaje de PSAl se asocia

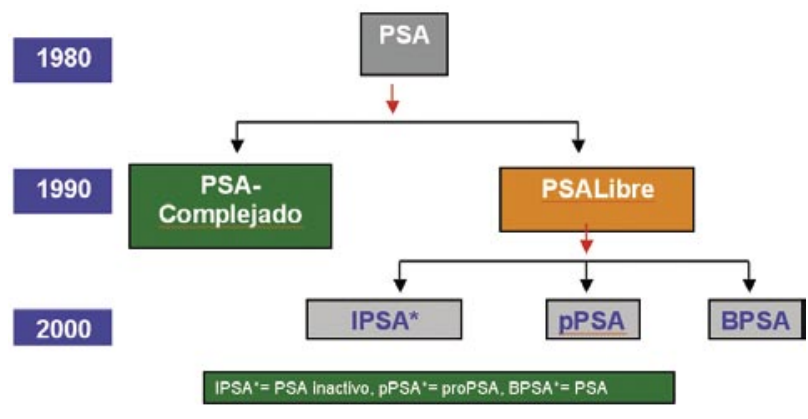

FIGURA 1. Evolución Histórica del PSA y sus Isoformas. a un mayor riesgo de cáncer. Diferentes estudios 1 $(19,22-25)$ han demostrado la validez de este ratio $\mathrm{PSAl} / \mathrm{t}$ en este rango o incluso para valores de PSA entre $2.5-4 \mathrm{ng} / \mathrm{ml}$ (Tabla II) si bien no existe un consenso total sobre el punto de corte a utilizar por los resultados dispares de los trabajos. Algunas de las series son procedentes de poblaciones de estudio y metodología con biópsias diferentes lo dificulta la interpretación aunque en general se acepta un valor del $25 \%$ como referencia aconsejable. El trabajo de Catalona (21) junto con otras series posteriores validan el PSAl y sus ratios también para este rango de $2.5-4 \mathrm{ng} / \mathrm{ml}$.

Por otra parte el PSAI en especial el ratio de PSAl/ $t$ ha sido estudiado en la capacidad para definir riesgo de agresividad tumoral, demostrando que especialmente en los porcentajes inferiores al $15 \%$ del PSAl puede ser un buen indicador (26). Así mismo también queda bien definido el riesgo creciente de posibilidad de tumor dependiendo del ratio $\% \mathrm{PSAl} / \mathrm{t}$ así pacientes con un ratio menor del 10\% su posibilidad de diagnosticar tumor se multiplica por dos (21).

\section{PSA Complejado}

El PSAc forma parte del $70 \%$ a $90 \%$ del PSA circulante. El grupo escandinavo $(10,14)$ a inicios de los 90 define el PSAc pero sin embargo su desarrollo como test y aprobación de la FDA no fueron parejas a la del PSAl. Tal y como se expresa en la tabla al igual que como sucedió con el PSAl diferentes trabajos han demostrado la validez de esta forma de PSA para los rangos de 4-10 y menores de 4 (27-32) (Tablas I y II). Brawer (27) es el primero en demostrar en su estudio que el PSAc tiene unos valores de sensibilidad y especificidad mayores que el PSA total y equivalentes a los del ratio PSAl/total indicando que el rango 4-10, puede plantearse como un test único. Otro estudio desarrollado de forma multicéntrica por Partin (32) con 831 pacientes estudiados con PSA $<10$ validan de nuevo la especificad y sensibilidad del PSAc sobre el total y no encuentra diferencias sobre el valor de los ratios de PSA con el PSAc por lo que se considera igualmente como test único proponiendo los puntos de 3,2-8,3ng/ml para el equivalente del PSAt $4-10 \mathrm{ng} / \mathrm{ml}$ y $2,2 \mathrm{ng} / \mathrm{ml}$ para el PSAc en rango de $2,5 \mathrm{ng} / \mathrm{ml}$ del PSAt. En el estudio realizado por nuestro grupo el PSAc con 1055 pacientes estudiados con primeras biopsias y rango de PSA 4-10, demostró igualmente mayor capacidad predictiva que el PSAt con diferencias significativas en el área bajo la curva siendo para el PSAt 0.59 y para el PSAc 0.63 no existiendo diferencias entre los ratios $\mathrm{PSAl} / \mathrm{c}$ y con un mayor número de biopsias ahorradas (30). (Figura 2). 


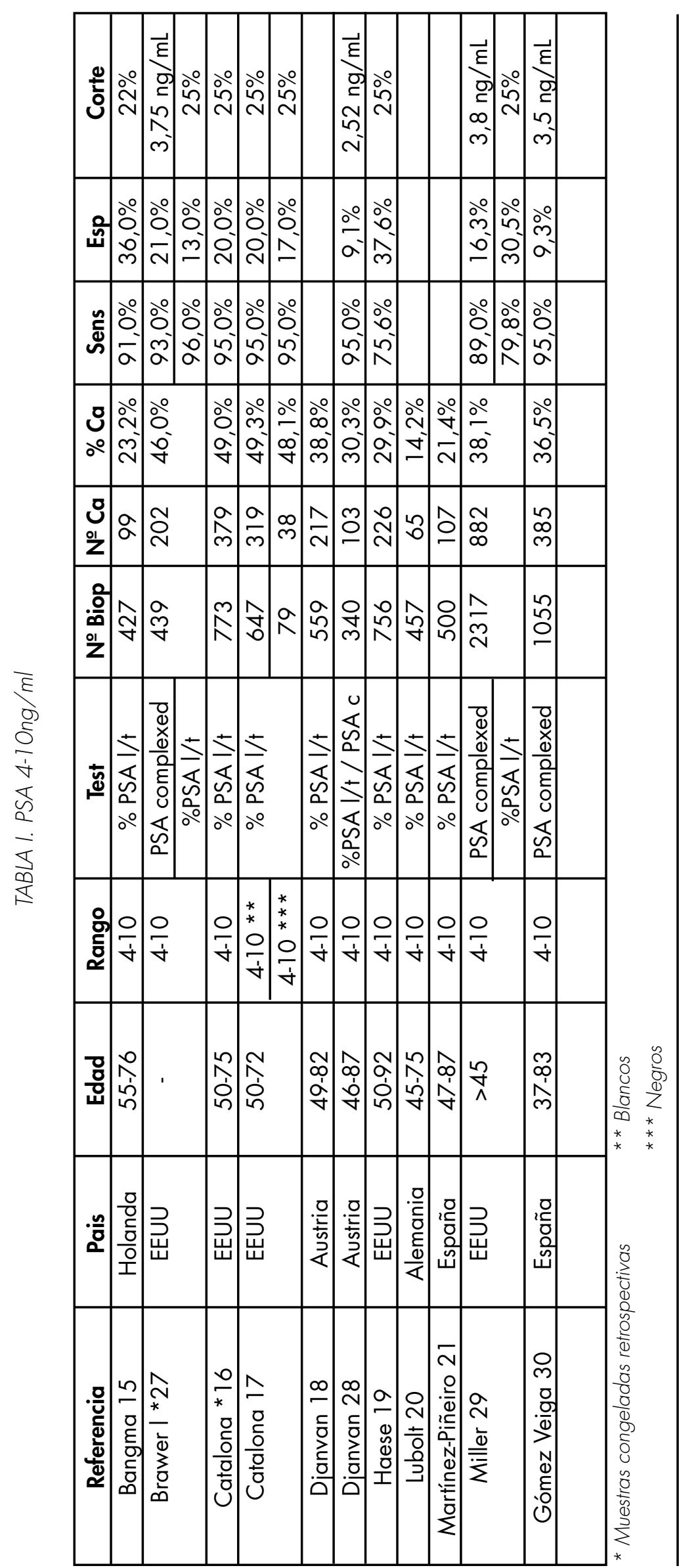

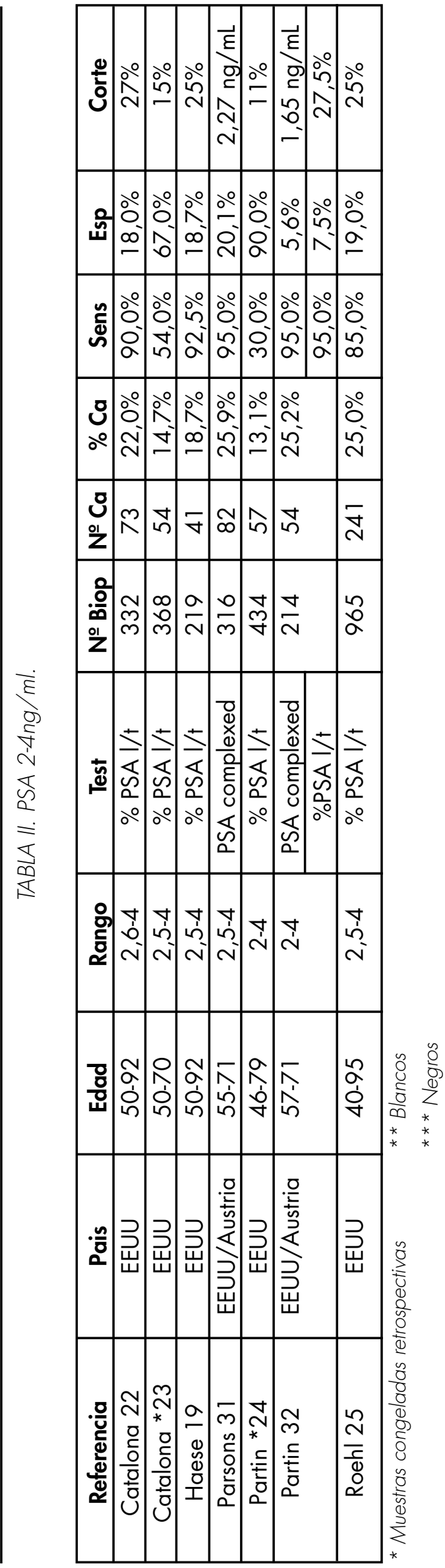




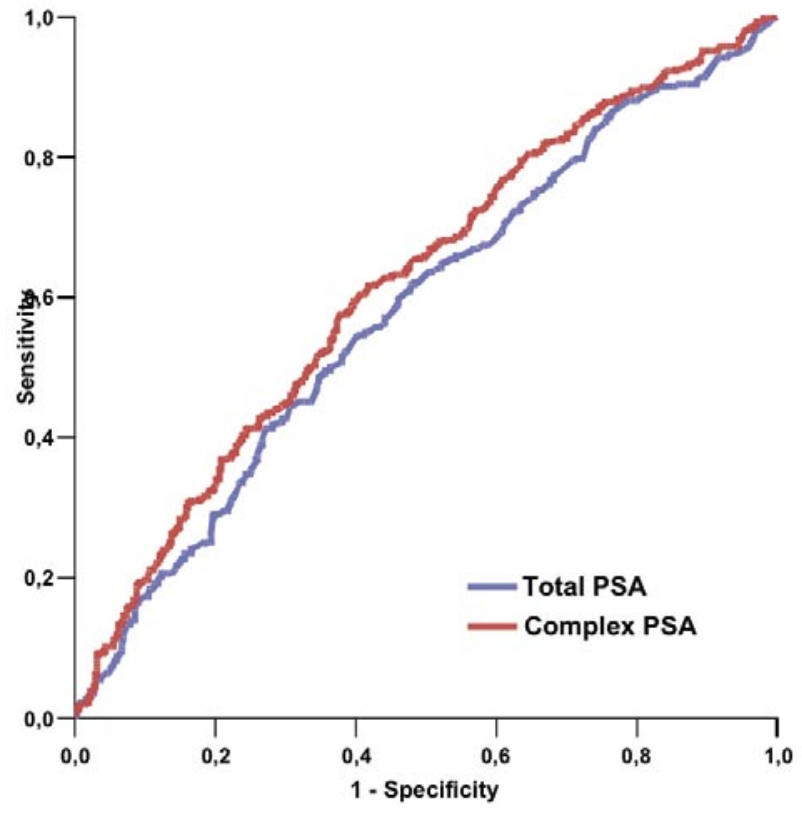

FIGURA 2. Curva ROC del PSAt y PSAc (n=1055). Área bajo la curva 0,58 y 0,63 para PSAt y PSAc respectivamente, $p<0,001$.

En este mismo contesto algunos trabajos han puesto en cuestión la validez de este test para rangos entre $2.5-4 \mathrm{ng} / \mathrm{ml}(33)$, por el contrario otros autores han reafirmado la validez para este mismo rango $(34,35)$. Es posible que alguno de estas discordancias tenga que ver con diferencias en los subgrupos de pacientes estudiados así como el propio test utilizado.

Interesante el análisis coste-beneficio de Babaian (36) y Ellison (37) que con la utilización del PSAc tanto para despistaje sistemático o precoz la cantidad de dinero ahorrados sería del orden $38 \mathrm{mi}$ llones de dólares americanos por millón de pacientes que se realice el PSA. En general y globalmente todos los trabajos demuestran una mejor selección de pacientes en particular en los rangos de PSA 4-10 con el PSAc.

\section{PSA $<4$}

Uno de los puntos a debate en el momento actual es el punto de corte para el PSA y definir el momento de hacer la biopsia. Si bien el valor de PSA en $4-10 \mathrm{ng} / \mathrm{ml}$ ha sido el valor estándar hasta la actualidad, dados los resultados de diferentes trabajos y muy en particular los relacionados con el estudio PTCP (38) ponen a debate dicha cuestión.

La revisión de Catalona (22) de 1997 es la primera en que señala y pone en tela de juicio el nivel del PSA para realizar las biopsias. Este trabajo hecho sobre una población de despistaje sistemático obtiene un $22 \%$ de biopsias positivas de 332 pacientes biopsiados con PSA entre 2,6-4ng/ $\mathrm{ml}$ siendo estos tumores un $82 \%$ localizados. Más recientemente y con un volumen importante de pacientes estudiados, 2950 en el estudio de PTCP (38) en 449 se detecta cáncer con un porcentaje de $12.5 \%$ para el rango de $0.5 \mathrm{ng} / \mathrm{ml}$ a $26.9 \%$ para el rango de $3.1-4$ $\mathrm{ng} / \mathrm{ml}$, siendo en este último el $25 \%$ de los tumores de alto grado. La agresividad en el incremento del PSA se confirmarían en la extensión de este estudio sobre pacientes operados con prostatectomía radical y PSA de menos de $3 \mathrm{ng} / \mathrm{ml}$. Otro estudio derivado del despistaje sistemático europeo el "ERSPC" de 883 pacientes con PSA entre 2-3.9 $\mathrm{ng} / \mathrm{ml}$ detectan 126 tumores (14.2\%) destacando en el rango de 3$3,9 \mathrm{ng} / \mathrm{ml}$ un $19,8 \%$ de positivos (39). Los datos de otros trabajos referidos a población japonesa obtienen porcentajes del $26,3 \%$ de biopsias positivas en dicho rango, siendo el Gleason 7 o más de un 19\% de estos tumores (34). Si bien parte de estos estudios desde el punto de vista metodológico son diferentes (despistajes sistemático, precoz, forma de biopsia) con diferentes metodologías todos ellos muestran un volumen importante de tumores. De ello se desprenden grandes dudas sobre la conveniencia de rebajar los niveles de PSA. Esto traería consecuencias evidentes. De una parte aumentaríamos de forma significativa el número de biopsias a realizar con importantes repercusiones socio-económicas y segundo que probablemente detectaríamos o tendríamos la duda de si estaríamos aumentando las sobre-detección con tumores insignificantes.

Los datos de estudios de screening en el futuro podrían aclararnos algunos de estos puntos, si bien en la actualidad podemos pensar que en pacientes con expectativa de vida mayor antecedentes familiares de tumor los riesgos/beneficios de realizar biopsia en estos rangos se deben debatir con el paciente y en cualquier caso no generalizar la práctica de la biopsia $(40,41)$.

\section{VALOR DEL PSA EN EL DESPISTAJE SISTEMÁ- TICO}

Uno de los mayores retos que presenta el PSA en las últimas décadas es la validez del mismo como método de despistaje sistemático. La finalidad del mismo se plantea en la investigación de una enfermedad con alta prevalencia (primer tumor en varones) y de tener una utilidad clínica para la enfermedad y pacientes en un valor de costes sostenibles. El test es sencillo y en la actualidad barato, hecho que favorece su desarrollo para la validez como método de 
despistaje. Dos grandes estudios están en marcha, el europeo "European Randomised Study of Screening for Prostate Cancer" "ERSPC" con más de 183.000 pacientes incluidos, entre 50 y 74 años. El otro gran estudio el que desarrollan en EEUU "The Prostate, Lung and Colorectal and Ovarian cancer screening trial" "PLCO" con 27.000 varones de edades entre 55 y 74 años. Ambos en marcha, sus datos no los obtendremos probablemente hasta 2009 o 2010.

Algunos estudios ya han puesto de manifiesto la posibilidad y la ventaja del screening. El estudio Canadiense con 46.000 pacientes demuestra una mejoría clara en la mortalidad con 15 muertes por año por cada 100.000 varones en los sometidos a screening sobre 49 por cada 100.000 habitantes pacientes fallecidos en los que no se realizó dicho screening (42). El otro de los estudios que igualmente demuestra una mejoría en la supervivencia ha sido el desarrollado en Austria en concreto en el área del Tirol (43). Ambos estudios aunque demuestran resultados claramente favorables son metodológicamente cuestionables dadas muchas de las interrogantes en la forma y desarrollo de los mismos, por lo tanto es muy posible que hasta que los estudios en EEUU y Europa concluyan no podamos obtener datos de certeza.

Múltiples factores pueden influir en el resultado de estos grandes estudios, en los que las diferencias pretenden ver si existe una mejoría en mortalidad específica en pacientes sometidos a control con PSA sobre aquellos que no han sido controlados. Algunos de los sesgos pueden venir dados por la propia selección, el adelanto a un diagnóstico, o el fenómeno de alargar el diagnóstico previo y por lo tanto definir la mejoría. El límite del PSA, en muchos de los casos ha sido otro de los factores cambiante, menor de 4 mayor o igual a 4, pacientes perdidos, frecuencia de revisiones, técnicas de biopsia (44). Muchos de estos factores pueden estar alterando los resultados y provocando una contaminación indirecta o directa, si bien estos han sido desarrollados para asumir un nivel de hasta un $20 \%$ de contaminación. En todo caso alguno de los datos recogidos del desarrollo en la actualidad del screening del PLCO pueden ser preocupantes puesto que el volumen de sobre-diagnóstico se estima entre un $29 \%$ a $44 \%$ lo cual nos lleva a una profunda reflexión (45).

En cualquier caso otros datos indirectos son valorados positivamente. El estudio escandinavo (46), publicado recientemente, demuestra una reducción en la mortalidad específica siendo del $9,6 \%$ en los pacientes que habían sido operados frente a un 14,9\% de los que optaron por esperar y ver, y un sub-análisis de este mismo estudio señala como los pacientes de menos de 65 años operados el riesgo de morir por cáncer de próstata se reduce un $57,8 \%$, hecho muy relevante puesto que precisamente a la población joven es hacia la que debe ir dirigido el despistaje sistemático. De otra manera y aún con todas las incógnitas que ello plantea, los datos publicados y contenidos específicamente sobre las estadísticas de mortalidad en EEUU demuestran como de forma paulatina y progresiva, la mortalidad específica está disminuyendo; todo ello y como se comentó a pesar de estar los estudios en marcha, pueden ser índices de que el despistaje sistemático puede mejorar la supervivencia (5).

\section{MODELOS PREDICTIVOS}

El valor del PSA, especialmente en este rango de 4-10 ng/ml, la posibilidad de detectar un tumor no ocupa un área bajo la curva de más de $60 \%$, asumiendo que si tiráramos una moneda al aire en el $60 \%$ de los casos acertaríamos y en el $40 \%$ de los casos falláramos. Las áreas comprendidas entre los valores del el $80 \%$ aportan más especificidad a las pruebas. Así, edad, volumen, volumen ajustado al área transicional, densidad de PSA, han contribuido a la mejoría de la especificidad dependiendo del punto de corte utilizado (47-49). Los nomogramas en sus diferentes formas (regresión logística, redes neuronales, árboles de decisión) contribuyen a aumentar la especificidad en todos los niveles, muy en particular en las zonas grises de PSA $4-10 \mathrm{ng} / \mathrm{ml}$; así en diferentes experiencias, los posibles factores claramente influyentes o discriminantes en la presencia de tumor, pueden ser utilizados para la toma de decisiones. Dichas herramientas contribuyen en muchos casos a disminuir la incertidumbre y por lo tanto a aumentar la exactitud diagnóstica. En un trabajo de nuestro servicio (50) en 2683 primeras biopsias, utilizando la regresión logística, con variables como edad, volumen, \%PSAl/t, tacto rectal el área bajo la curva para el PSA tenía un valor de 0.67 y con el modelo de regresión logística obteníamos una curva de 0.80 $(p<0.001)$ siendo las diferencias significativas entre grupos facilitando el cálculo de probabilidades.

La utilización de las redes neuronales o árboles de decisión son algunos de los métodos más complejos y utilizados para estos cálculos, la finalidad es la misma, si bien pueden ser elementos complementarios, no tienen por que ser los resultados específicamente diferentes al modelo clásico de la regresión logística. En un análisis de 1986 biopsias utilizando un grupo control de 689 pacientes, el área bajo la curva para la red neuronal era de 0.78 frente a un 0.80 para el modelo de regresión logística, no existiendo diferencias significativas entre ambos 
métodos (51). Las conclusiones que obtiene Garzotto (52) en el estudio de 1433 pacientes y con un PSA de menos de 10, utilizando los árboles de decisiones y regresión logísticas, son las mismas, con áreas bajo la curva de 0.74 para la regresión logística frente al 0.72 para los árboles de decisiones. Es interesante la posibilidad de desarrollar modelos no solo que predigan tumor si no agresividad tumoral. En este sentido se desarrollan nuevas experiencias (41).

En conclusión, los modelos nos ayudan pero no debemos olvidar que son posibilidades que nos aproximan para tomar decisiones con el paciente, que por otra parte están sujetas a posibles defectos de validación de los mismos, no siempre superponibles a nuestras experiencias por sesgos de población, técnica o muestreo.

\section{FACTORES RELACIONADOS CON LAS MODI- FICACIONES DEL PSA}

El PSA se ha relacionado con múltiples factores que pueden provocar modificaciones del mismo, algunos de ellos como (volumen, procesos inflamatorios, actividad física, edad, tratamientos, exploraciones o variaciones en el propio test etc). Por otro lado la propia cinética del mismo no es bien conocida y se asocia a modificaciones progresivas aunque no bien aclaradas $(53,54)$.

Volumen: la relación entre el volumen y el incremento del PSA fue puesta de manifiesto por Benson (55) en el 92, deja claro que a mayor volumen prostático mayor producción de PSA. Este autor apoyado en sus estudios desarrolla el concepto de densidad de PSA con el que podría seleccionar pacientes posteriormente en rangos de PSA 4-10 $\mathrm{ng} / \mathrm{ml}$. Estos estudios han tenido revisiones en otros y no se confirmó el hecho de que la densidad del PSA per se fuese más específica $(56,57)$. Esto se puede explicar por el hecho de que la contribución del PSA producido por un tumor independientemente del tamaño puede ser variable de forma que un mismo tumor sobre una lesión más pequeña pudiera ser diagnosticado o no. Algunos trabajos han revisado la utilidad de los volúmenes de áreas transicionales y ajustarlos al desarrollo de modelos suponiendo en dichos modelos un incremento de la sensibilidad (28). Es el volumen sin duda un factor relacionado con la producción de PSA pero no debe de integrarse como único parámetro (58).

Edad: Oesterling (59) relacionó por primera vez la importancia de la edad como factor con el PSA. Encuentra en su estudio de 2.219 varones dicha relación y recomienda cortes por edades de valores de PSA que irían para los 50 a 59 años de $3,5 \mathrm{ng} / \mathrm{ml}$ y para los de $70-79$ de $6,5 \mathrm{ng} / \mathrm{ml}$, lo cual serviría para la reducción de biopsias. Más recientemente e insistiendo en este tema Punglia (60) hace una revisión sobre el efecto de la edad y la presencia de la hiperplasia con el PSA y desarrolla un modelo que confirma la importancia de este factor para la toma de decisiones muy especialmente en los rangos de PSA $<4$.

Inflamación: La elevación del PSA puede también ser causa de elevaciones en muchos casos exponenciales como la situaciones de prostatitis aguda. Por otra parte muchas de las biopsias demuestran datos de inflamación. El efecto de esta sobre PSA ha sido estudiada $(61,62)$. En general estas revisiones no demuestran diferencias significativas, en los valores del PSA entre pacientes con HBP permaneciendo estables los valores en la patología benigna, incluso en los ratios de porcentajes de PSAl y total, por lo que se concluye que puede discriminar bien pacientes para biopsiar especialmente con el \%PSAl/t.

Tratamientos: Algunos de los tratamientos y en particular aquellos que alteran la modificación de la dehidrotetosterona como los derivados de la 5 areductasa han sido estudiados en el contexto de ensayos clínicos. Tanto Finasteride como Dutesteride se ha desmostrado que tienen una reducción aproximada del $50 \%$ de los valores del PSA, reducción que en general se establece en el período del año si bien a los 6 meses hay una reducción significativa próxima a ese valor. En el seguimiento de estos pacientes hemos de corregir el dato del PSA multiplicándolo por 2 (63). La problemática se plantea en cualquier caso con los ratios de PSA para definir riesgo, especialmente en pacientes con PSA inicial $<4$ o como definir los incrementos una vez que el paciente haya podido dejar el tratamiento.

Cinética del PSA: Carter (64) fue el primero en demostrar como los valores de PSA por encima de $0.75 \mathrm{ng} /$ $\mathrm{ml}$ fue significativamente mayores en los pacientes que presentaban cáncer sobre aquellos que tenían patología benigna. Más recientemente D'Amico (65) correlaciona los incrementos de PSA anuales en 1100 varones y demuestra como los incrementos de más de $2 \mathrm{ng} / \mathrm{ml}$ se relacionan con mayor riesgo de muerte y lógicamente con mayor riesgo de tumores agresivos. Datos ellos muy importantes para la toma de decisiones. También los datos del estudio de screening europeo se comprueba como la velocidad e incremento del PSA en los pacientes con tumor es diferente a los que tienen patología benigna pero inferior a $0.75 \mathrm{ng} / \mathrm{ml} / \mathrm{año}$ con cifras de $0.62 \mathrm{ng} / \mathrm{ml} /$ año frente a $0.46 \mathrm{ng} / \mathrm{ml} /$ año para los que tienen o no tienen tumor (66). La velocidad sin duda es un factor a considerar a la hora de biopsiar si bien tam- 
bién hemos de evaluar algunos factores limitantes. En esta valoración, son importantes las variaciones inter-individuales $(54,67)$ que incluyen la propia disminución del PSA en el seguimiento. Interesante en este punto el trabajo de Boddy (68) en el que analiza a 160 pacientes en los que repite PSA y en 21 (13\%) disminuye el mismo, de estos repite biopsia y en 5 $(4 \%)$ presentaban tumor. La propia variación individual llega a la reflexión a Eastham (69) en su trabajo sobre 972 pacientes de que una sola determinación no puede servirnos para la toma de decisiones. Entre otros datos su trabajo refleja como un elevado porcentaje de pacientes con PSA $>4(44 \%)$ y $50 \%$ en los que tienen PSA >2.5 no presentaban en estudios sucesivos elevaciones del PSA.

Otro punto asociado a las modificaciones del propio PSA es incluso el que representa la reproductividad y variabilidad entre ensayos, hecho que debemos de considerar a la hora de la toma de decisiones, así en algunos de los casos se ha demostrado como pueden existir variaciones de aproximadamente de más de un $20 \%$ entre diferentes determinaciones de ensayos $(70,71)$, lo cual es un punto crítico para decidir biopsias o rebiopsias en pacientes con rangos bajos de PSA, en conclusión si bien la velocidad es un factor importante en la toma de decisiones existen ciertas dudas que hacen que debamos de complementarlos con algunas otras variables.

\section{NUEVOS MARCADORES}

\section{Justificación}

La falta de especificidad del PSA nos lleva a plantearnos la necesidad de nuevos marcadores para mejorar la misma. El problema se plantea por dos hechos fundamentados. El primero es que aproximadamente 2 de cada 3 pacientes biopsiados no tienen tumor realizando por lo tanto biopsias innecesarias. Además muchos de los tumores que diagnosticamos son lesiones que van a tener un potencial no agresivo y por lo tanto podemos caer en la sobre-detección. Recientemente algunas voces de reconocido prestigio como el propio Stamey (72) han puesto en cuestión la validez del PSA actualmente como elemento diagnóstico. En su revisión con 1317 pacientes no encuentra correlación entre el PSA y el volumen tumoral y concluye que el PSA no es clínicamente útil en la actualidad para conocer el potencial tumoral, este hecho se contrapone en lo descrito previamente en su artículo que marcó el inicio de la era del PSA en donde aclaraba que dicho marcador era importante para la discriminación de potencial agresivo.

Este argumento en gran parte ha sido contestado en el trabajo del PTCP (38) donde se confir- ma una correlación clara entre incremento de PSA y proporción mayor de tumores de alto grado. Por otro lado el trabajo del grupo Freedland (74) en su revisión de 2.312 pacientes sometidos a prostatectomía radical con más de 1.900 pacientes con PSA $<10 \mathrm{ng} / \mathrm{ml}$ analiza entre otros el papel del PSA como factor para la predicción del grado de agresividad tumoral y factores adversos en el seguimiento, concluyendo que el PSA es un factor fundamental para la predicción del grado en su análisis uni-multivariado. En cualquier caso aunque como el propio Freedland reconoce "el PSA está vivo y bien", parece necesario la búsqueda de unos nuevos marcadores para mejorar la especificidad. La investigación de los nuevos marcadores en todo caso es difícil y para considerar efectivo uno nuevo ha de demostrar previamente una eficacia contrastada, que sea fácilmente detectable especialmente en suero, orina y fluidos y capaz de discriminar bien entre lesiones benignas o malignas. Es posible también que un sólo marcador por si mismo no sea capaz de explicar todas las condiciones pero en muchos de los casos pueden ser combinaciones de los mismos las que nos podrían ayudar. La lista de potenciales nuevos marcadores en la actualidad es interminable $(75,76)$. El ideal podría ser una proteína específica para el tumor. Las nuevas tecnologías aplicadas fundamentalmente en el capítulo de la biología molecular genómica, proteómica - bioinformática hacen tener fundadas esperanzas en que puedan facilitarnos la aparición de nuevos marcadores claramente más específicos.

\section{BIOLOGÍA MOLECULAR}

Uno de los trabajos más recientes e impactantes ha sido el publicado por Wang (77) en el que se caracteriza y se analiza la expresión de DNA recombinante (SEREX) y demuestran como las características específicas de 22 proteínas de estos tumores pueden ser reconocidas con dicha técnica. Con 119 pacientes con cáncer y 118 controles los resultados que consiguen, en particular para pacientes con PSA entre $4-10 \mathrm{ng} / \mathrm{ml}$, eleva la especificidad al $88 \%$, con una sensibilidad de $81,6 \%$ con un área bajo la curva del 0.93 siendo la misma curva para el PSA de sólo 0.56 . Cuando los mismos datos se utilizan para los rangos de PSA $2.5 \mathrm{ng} / \mathrm{ml}$ el área bajo la curva para dicho modelo es de 0.94 frente a un 0.50 para el PSA. Los resultados francamente impactantes deberán de ser en todo caso validados por series más amplias y reproducir la viabilidad de la técnica.

Otro de los grandes campos dentro de la biología molecular está centrado en el avance del campo de la metilación y los marcadores de la metilación. La $\alpha$-metilacil-coenzima A racemasa (AMACR) 
es una enzima localizada en el gen 5 y que juega un papel importante en la oxidación de cadenas de ácido graso. El gen regulador de dichas enzimas se ha demostrado tener una sobre-expresión muy específica dentro del cáncer de próstata en particular en el epitelio de células basales. El gran avance ha sido el desarrollo por parte de Rubin (78) demostrar la sobre-expresión de esta proteína en los en un estudio con 116 tumores y 108 con hiperplasia y con técnica de RT-PCR el AMACR se sobre-expresó en 84 piezas de biopsia prostática demostrando una sensibilidad de $97 \%$ y un $100 \%$ de especificidad para detectar cáncer. La consecuencia de este estudio más interesante es el desarrollo del trabajo y la validación en suero del AMACR como método para diferenciar tumores. Utilizando 32 sueros diferentes y 28 controles asignados de un grupo de 155 tumores y 259 controles fueron sometidos a estudio inmunológico con ELISA para AMACR; los resultados son importantes pues en el rango de $4-10 \mathrm{ng} / \mathrm{ml}$ de PSA, fue mayor la sensibilidad y especificidad $77,8 \%$ y $86 \%$ sobre $45 \%$ y $50 \%$ con áreas bajo la curva de 0.78 versus 0.49 ( $p<0.01$ ) en relación con el PSA (79). En todo caso se plantean problemas similares a los anteriormente citados en relación con la reproducibilidad de técnica y de estudios complementarios que confirmen estos datos.

Otro grupo de interés es el formado por la familia de los glutation-transferasas (GSTP1) cuya función básicamente es proteger a las células de la oxidación. Este marcador ha visto reducido su expresión en tumores de próstata. Con técnicas de metilación específica para PCR se ha logrado analizar (80). Otros estudios utilizando la orina tras masaje prostático, han demostrado una buna sensibilidad para detectar cáncer. En el $73 \%$ de los tumores se detectaba el marcador frente a un $2 \%$ en los casos de hiperplasia benigna prostática (81).

\section{Telomerasas}

La telomerasa, enzima esencial para la protección del DNA, ha sido propuesta como otro marcador tumoral. Un trabajo de 30 pacientes analiza la telomerasa en orina, encontrando en $100 \%$ de los tumores presente una actividad importante de dicha enzima, por el contrario en la hipertrofia benigna de próstata el $70 \%$ no presentaban dicha actividad y cuando presentaba actividad, valorada por método cuantitativo ésta era baja. Estos tumores, un 18\%, además presentaban una actividad muy elevada (82). Recientemente el grupo español de Dasi (83) estudia en suero el potencial de la telomerasa por técnica de PCR en suero en 68 pacientes y demuestra diferencias significativas en la expresión de la actividad entre los pacientes con cáncer y patología benigna incluido la prostatitis, con un nivel de especificidad del $60 \%$. Ambos estudios implican un nuevo camino si bien uno de los problemas que podría plantearse por la vía de la telomerasa puede ser la falta de especificidad como único marcador dada su presencia para otros tumores.

\section{HER2/nue}

Dentro de los factores de crecimiento el HER2/nue ha sido estudiado como factor de sobreexpresión en varios tumores incluyendo la mama. Recientemente se presentan trabajos que relacionan este marcador con los tumores de próstata. El trabajo de Osman (84) en suero y con inmunoanálisis analiza diferentes grupos de tumor y pacientes sin tumor. La conclusión del trabajo es que se encontraron cifras elevadas de dicho marcador claramente significativas entre los pacientes que se presentaban con tumor avanzado metastático y entre los que o no presentaban tumor o el tumor era localizado. El trabajo de Okegawa (85) analiza en 197 pacientes con cáncer en diferentes estadios y también pone de manifiesto el valor incrementado del marcador especialmente con enfermedad avanzada y metastásica sobre aquellos que tenían tumor localizado. Ambos trabajos ponen también de interés la utilización de un marcador comercializado y que se puede utilizar como posible factor pronóstico independiente. En el fututo habrá que valorarlo como marcador específico o de apoyo para la toma de decisiones.

\section{Proteómica}

La espectrometría aplicada a los nuevos marcadores entra en este momento en un punto de gran desarrollo, fruto de dicho desarrollo dos premios Nóbel han sido nombrados recientemente. La aplicación de esta sofisticada pero cada vez más estandarizada tecnología nos permite el conocimiento más definido de los perfiles protéicos de los tejidos y por lo tanto definir mejor posibles marcadores para tumor. En Europa un nuevo proyecto en marcha elabora diferentes estudios con la tecnología SELDI para estudiar en suero y orina nuevos patrones de perfil protéico (86). Diferentes trabajos han utilizado ya esta tecnología y así en el trabajo de Ornestein (87) estudio 1154 pacientes con PSA entre 2.5-10 con tumor y sin tumor validando un patrón para un algoritmo diagnóstico en el método utilizado con dicho patrón obtiene un $100 \%$ de sensibilidad y un $67 \%$ de especificidad consiguiendo con dicho modelo que el $67 \%$ de los pacientes no precisaran ser biopsiados. Otro trabajo utilizando otro patrón con metodología similar obtiene cifras de $85 \%$ de sensibilidad y $85 \%$ de especificidad para detectar tumores (88). El trabajo del grupo de $\mathrm{Li}$ (89) en esta misma línea con 246 pacientes 
analizados selecciona un panel de tres proteinas, cuando se compara el patrón PSA la especificidad es significativamente mejor para el patrón protéico con áreas bajo la curva de 0.54 frente a 0.64 . En conclusión la proteómica igualmente puede jugar un papel muy importante en el desarrollo de los nuevos marcadores.

\section{MARCADORES GENÉTICOS}

La diferente prevalencia de estos tumores en algunas razas o etnias han explicado la posibilidad de posibles factores familiares genéticos relacionados con el mismo. Aunque algunos tumores tienen relación familiar el porcentaje de los mismos en todo caso es bajo. Algunos genes como el HPCEl y el HPCE2 han sido estudiados entre grupos de riesgo y ambos se han relacionado con la vía de la producción del metabolismo androgénico y con un mayor riesgo de tumor. El CYB 17 cuyo mecanismo estaría ligado al riesgo también de desarrollar tumores parece debido a la producción elevada de testosterona. El CPY34A asociado con el citocromo P450 hepático y ligado a la hiperproducción de andrógenos ha sido otro de los marcadores que se ha visto relacionado con la producción de tumores $(86,90)$. En cualquier caso estos marcadores no parece que puedan tener una gran relevancia actual para el diagnóstico.

\section{KALICREINAS}

El descubrimiento de las kalicreinas en 1992 ha desarrollado la posibilidad también de nuevos marcadores. En total hay descritos quince tipos de kalicreinas (91). De todos ellos la kalicreina 2 que comparte un $80 \%$ de la estructura de la kalicreina 3 del PSA es la más estudiada. Otras kalicreinas como la HK6, HKO o HK 11 entran en estudios como también posibles marcadores (92). Los niveles de HK2 en estudios recientes apoyan la utilización del mismo en combinación con el PSA y sus isoformas, en particular con el PSA libre especialmente en rango de PSA 2-10ng/ml. Partin (24) en su estudio con 937 pacientes con rango de PSA 2-10 ng/ml obtiene un valor predictivo para detectar cáncer del $62 \%$ utilizando los ratios de HK2 y PSA libre. En las concentraciones de PSA 2-4ng/ml el ratio HK2/PSA libre el valor es del $40 \%$ con un ahorro significativo de biopsias. Steuber (93) analiza 416 pacientes, 252 con cáncer y estudia el PSA libre y las subfracciones del mismos incluyendo el HK2 y los correlaciona con los volúmenes referidos a la presencia de tejido tumoral o benigno. Comprueba que el HK2 junto las subfracciones del PSA libre se correlaciona bien con dicho volumen específico tumoral. Aunque con posibilidad de uso nos plantea dudas sobre las posibilidades de este marcador por el momento.

\section{NUEVAS ISOFORMAS DEL PSA}

La presencia de un mayor porcentaje de PSA libre en pacientes con hiperplasia benigna prostática llevó a valorar la posibilidad de estudiar marcadores específicos para las diferentes zonas de la próstata. Así generalmente el área transicional está más relacionada con la hiperplasia y la periférica con el cáncer, podrían pues definir mejor las poblaciones de riesgo . Dicha asociación fue puesta de manifiesto por Mikolajczyk (94) en su estudio donde demuestra sobre próstatas de más 50 grs o menos de 25 grs como lo que sería el proPSA en el $72 \%$ de los casos no estaba presente en el área transicional. Una evolución de este mismo grupo fue el desarrollo de un inmunoanálisis que pueda diferenciar el bPSA que es responsable del 15 a $50 \%$ del PSA libre y el proPSA (95). Catalona (96) recientemente en un trabajo valora el pPSA para diferentes rangos de PSA 2-4 y 4-10 con un total de 1091 pacientes. Los datos demuestran que entre rangos el pPSA por si mismo no discrimina mejor que el propio PSAt, PSAI o PSAc pero cuando se utiliza el ratio el porcentaje PSAI/pPSA estos datos se hacen significativos. Si bien esta información concluye con diferencias importantes entre el PSA y PSAc, metodoló gicamente no se utiliza en todos los pacientes analizados el PSAc, por lo que se puede pensar que aunque puedan tener validez deberían realizarse más estudios que confirmen estos datos prometedores pero que hoy por hoy hacen difícil su exacta dimensión.

\section{CONCLUSIONES}

El PSA es el mejor marcador biológico que tenemos en la actualidad para el diagnóstico de cáncer de próstata. En su evolución desde hace más de veinticinco años nos ha facilitado el diagnóstico, siendo en la actualidad más del $90 \%$ de los tumores localizados y con datos de descenso de mortalidad especialmente en USA, lo que refleja en cierta medida que dicho diagnóstico temprano de la enfermedad puede ser efectivo.

La evolución y mejor conocimiento del marcador, nos ha facilitado con el paso del tiempo a través de las isoformas libre y complejada, la aplicación de factores relacionados con la producción de PSA, nos han mejorado la selección de pacientes y por consiguiente la especificidad

Todo parece indicar que el hasta ahora clásico corte de PSA en $4 \mathrm{ng} / \mathrm{ml}$ no parece ser el 
adecuado especialmente en pacientes jóvenes y con expectativa de vida mayor, debiendo reducirse posiblemente al nivel a $2.5 \mathrm{ng} / \mathrm{ml} \circ 2.2 \mathrm{ng} / \mathrm{ml}$ para la forma complejada.

Las datos de despistaje sistemático que están en marcha es posible que nos aclaren alguno de estos puntos, mientras tanto es recomendable el seguir informando a los pacientes de los riesgos beneficios de estas decisiones.

En el horizonte especialmente con todo lo que implica el desarrollo de la biología molecular en su diferentes facetas nos pueda facilitar en el futuro próximo nuevos marcadores.

En este momento el PSA sigue siendo el marcador estándar para el diagnóstico del cáncer de próstata.

\section{BIBLIOGRAFÍA y LECTURAS RECOMENDADAS ( ${ }^{*}$ lectura de interés $y^{* *}$ lectura fundamental)}

**1. WANG, M.C.; VALENZUELA, L.A.; MURPHY, G.P. y cols.: "Purificacion of an human prostate specific antigen". Invest. Urol., 17: 159, 1979.

2. HARA, M.; KOYANAGI, Y.; INOUE, T. y cols.: "Some physico-chemical characteristics of "gammaseminoprotein," an antigenic component specific for human seminal plasma. Forensic immunological study of body fluids and secretion". VII Nippon Hoigaku Zasshi, 25: 322, 1971.

3. DENMEADE, S.R.; ISAACS, J.T.: "A history of prostate cancer treatment". Nat. Rev., 2: 389, 2002.

*4. HODGE, K.K.; McNEAL, J.E.; TERRIS, M.K. y cols.: "Random systematic versus directed ultrasound guided transrectal core biopsies of the prostate". J. Urol., 142: 71, 1989.

*5. JEMAL, A.; SIEGEL, R.; WARD, E. y cols.: "Cancer statistics, 2006”. C.A. Cancer J. Clin., 56: 106, 2006.

6. BOYLE, P.; FERLAY, J.: "Cancer incidence and mortality in Europe, 2004". Ann. Oncol., 16: 48, 2005.

7. Instituto Nacional de Estadística. Salud. Defunciones según la Causa de Muerte. Año 2004. [Internet]. Madrid: INE [acceso 7/11/2006]. Disponible en: http://www.ine.es/

8. KUNDU, S.D.; ROEHL, K.A.; EGGENER, S.E. y cols.: "Potency, continente and complications in 3,477 consecutive radical retropublic prostatectomies". J. Urol., 172: 2227, 2004.

9. YOUSEF, G.M.; DIAMANDIS, E.P.: "The new human tissue kallikrein gene family: structure, function, and association to disease". Endocr. Rev., 22: $184,2001$.
**10. LILJA, H.; CHRISTENSSON, A.; DAHLEN, U. y cols.: "Prostate specific antigen in serum occurs predominantly in complex with alpha-1 antichymotrypsin". Clin. Chem., 37: 1618, 1991.

*11. MIKOLAJCZK, S.D.; MILLAR, L.S.; WANG, T.J. y cols.: "A precursor form of prostate-specific antigen is more highly elevated in prostate cancer compared with bening transition zone prostate tissue". Cancer Res., 60: 756, 2000.

**12. STAMEY, T.A.; YANG, N.; HAY, A.R. y cols.: "Prostate-specific antigen as a serum marker for adenocarcinoma of the prostate". N. Engl. J. Med., 317: 909, 1987.

**13. CATALONA, W.J.; SMITH, D.S.; RATLIFF, T.L. y cols.: "Measurement of prostate-specific antigen in serum as a screening test for prostate cancer". N. Engl. J. Med., 324: 1156, 1991.

14. CHRISTENSSON, A.; BJORK, T.; NILSSON, O. y cols.: "Serum prostate specific antigen complexed to alpha 1-antichymotrypsin as an indicator of prostate cancer". J. Urol., 150: 100, 1993.

15. BANGMA, C.H.; RIETBERGEN, J.B.; KRANSE, R. y cols.: "The free-to-total prostate specific antigen ratio improves the specificity of prostate specific antigen in screening for prostate cancer in the general population”. J. Urol., 157: 2191, 1997.

**16. CATALONA, W.J.; PARTIN, A.W.; SLAWIN, K.M. y cols.: "Use of the percentage of free prostate-specific antigen to enhance differentiation of prostate cancer from benign prostatic disease: a prospective multicenter clinical trial". JAMA, 279: 1542, 1998.

17. CATALONA, W.J.; RAMOS, C.G.; CARVALHAL, G.F. y cols.: "Lowering PSA cutoffs to enhance detection of curable prostate cancer". Urology, 55: 791, 2000.

18. DJAVAN, B.; REMZI, M.; ZLOTTA, A.R. y cols.: "Combination and multivariate analysis of PSAbased parameters for prostate cancer prediction". Tech. Urol., 5: 71, 1999.

19. HAESE, A.; DWORSCHACK, R.T.; PARTIN, A.W.: "Percent free prostate specific antigen in the total prostate specific antigen 2 to $4 \mathrm{ng} / \mathrm{ml}$. range does not substantially increase the number of biopsies needed to detect clinically significant prostate cancer compared to the 4 to $10 \mathrm{ng} / \mathrm{ml}$ range". J. Urol., 168: 504, 2002.

20. LUBOLDT, H.J.; SWOBODA, A.; BORGERMANN, C. y cols.: "Early Detection Project Group Of the German Society of Urology. Clinical usfulness of free PSA in early detection of prostate cancer". Onkologie, 24: 33, 2001.

21. MARTÍNEZ-PIÑEIRO, L.; GARCÍA MEDIERO, J.M.; GONZÁLEZ GANCEDO, P. y cols.: "Probability of prostate cancer as a function of the percentage of free prostate-specific antigen in patients with a non-suspicious rectal examination and total prostate-specific antigen of 4-10 ng/ml”. World J. 
Urol., 22: 124, 2004.

**22. CATALONA, W.J.; SMITH, D.S.; ORNSTEIN, D.K.: "Prostate cancer detection in men with serum PSA concentrations of 2.6 to $4.0 \mathrm{ng} / \mathrm{mL}$ and benign prostate examination. Enhancement of specificity with free PSA measurements". JAMA, 277: 1452, 1997.

23. CATALONA, W.J.; PARTIN, A.W.; FINLAY, J.A. y cols.: "Use of percentage of free prostate-specific antigen to identify men at high risk of prostate cancer when PSA levels are 2.51 to $4 \mathrm{ng} / \mathrm{ml}$ and digital rectal examination is not suspicious for prostate cancer: an alternative model". Urology, 54: 220, 1999.

24. PARTIN, A.W.; CATALONA, W.J.; FINLAY, J.A. y cols.: "Use of human glandular kallikrein 2 for the detection of prostate cancer: preliminary analysis". Urology, 54: 839, 1999.

25. ROEHL, K.A.; ANTENOR, J.A.; CATALONA, W.J.: "Robustness of free prostate specific antigen measurements to reduce unnecessary biopsies in the 2.6 to $4.0 \mathrm{ng} / \mathrm{mL}$ range". J. Urol., 168: 922, 2002.

26. MOROTE, J.; ENCABO, G.; DE TORRES, I.M.: "Use of percent free prostate-specific antigen as a predictor of the pathological features of clinically localized prostate cancer". Eur. Urol., 38: 225, 2000.

*27. BRAWER, M.K.; CHELI, C.D.; NEAMAN, I.E. y cols.: "Complexed prostate specific antigen provides significant enhancement of specificity compared with total prostate specific antigen for detecting prostate cancer". J. Urol., 163: 1476, 2000.

28. DJAVAN, B.; REMZI, M.; ZLOTTA, A.R. y cols.: "Complexed prostate- specific antigen, complexed prostate-specific antigen density of total and transition zone, complexed/total prostate-specific antigen ratio, free-to-total prostate-specific antigen ratio, density of total and transition zone prostatespecific antigen: results of the prospective multicenter European trial". Urology, 60: 4, 2002.

29. MILLER, M.C.; O’DOWD, G.J.; PARTIN, A.W. y cols.: "Contemporary use of complexed PSA and calculated percent free PSA for early detection of prostate cancer: impact of changing disease demographics". Urology, 57: 1105, 2001.

30. GOMEZ VEIGA, F.; BARBAGELATA, A.; PONCE, J. y cols.: "Analysis of complex prostatic specific antigen and their ratios to detect prostate cancer undergoing a first biopsy with total PSA 410ngr/ml. A series of 1055 patients". Urology, 68: 273, 2006.

31. PARSONS, J.K.; BRAWER, M.K.; CHELI, C.D. y cols.: "Complexed prostate specific antigen (PSA) reduces unnecessary prostate biopsies in the 2.6$4.0 \mathrm{ng} / \mathrm{mL}$ range of total PSA". BJU Int., 94: 47, 2004.

32. PARTIN, A.W.; BRAWER, M.K.; BARTSCH, G. y cols.: "Complexed prostate specific antigen improves specificity for prostate cancer detection: results of a prospective multicenter clinical trial". J. Urol., 170: 1787, 2003.

33. LEIN, M.; KWIATKOWSKI, M.; SEMJONOW, A. y cols.: "A multicenter clinical trial on the use of complexed prostate specific antigen in low prostate specific antigen concentrations". J. Urol., 170: 1175, 2003.

34. KOBAYASHI, T.; KAMOTO, T.; NISHIZAWA, K. y cols.: "Prostate-specific antigen (PSA) complexed to a1-antichymotrypsin improves prostate cancer detection using total PSA in Japanese patients with total PSA levels of 2.0-4.0 ng/mL". BJU Int., 95: 761, 2005.

35. KELLER, T.; BUTZ, H.; LEIN, M. y cols.: "Discordance analysis characteristics as a new method to compare the diagnostic accuracy of tests: example of complexed versus total prostate-specific antigen". Clin. Chem., 51: 1, 2005.

36. BABAIAN, R.J.; NAYA, Y.; CHELI, C.D. y cols.: "The detection and potential economic value of complexed prostate specific antigen as a first line test”. J. Urol., 175: 897, 2006.

37. ELLISON, L.; CHELI, C.D.; BRIGHT, S. y cols.: "Cost-benefit analysis of total, free/total, and complexed prostate-specific antigen for prostate cancer screening". Urology, 60: 42, 2002.

**38. THOMPSON, I.M.; PAULER, D.K.; GOODMAN, P.J. y cols.: "Prevalence of prostate cancer among men with a prostate-specific antigen level $4 \leq$ ng per millilitre". N. Engl. J. Med., 350: 2239, 2004.

39. RAAIJMAKERS, R.; BLIJENBERG, B.G.; FINLAY, J.A. y cols.: "Prostate cancer detection in the prostate specific antien range of 2.0 to $3.9 \mathrm{ng} / \mathrm{ml}$ : value of percent free prostate specific antigen on tumor detection and tumor aggressiveness". J. Urol., 171: 2245, 2004.

40. NAM, R.K.; TOI, A.; TRACHTENBERG, J. y cols.: "Making sense of prostate specific antigen: improving its predictive value in patients undergoing prostate biopsy". J. Urol., 175: 489, 2006.

*41. THOMPSON, I.M.; ANKERST, D.P.; CHI, C. y cols.: "Assessing prostate cancer risk: results from the prostate cancer prevention trial". J. Natl. Cancer Inst., 98: 529, 2006.

42. LABRIE, F.; CANDAS, B.; DUPONT, A. y cols.: "Screening decreases prostate cancer death: first análisis of the 1988 Quebec prospective randomized controlled trial". Prostate, 38: 83, 1999.

*43. OBERAIGNER, W.; HORNINGER, W.; KLOCKER, H. y cols.: "Reduction of prostate cancer mortality in Tyrol, Austria, after introduction of prostate-specific antigen testing”. Am. J. Epidemiol., 164: 376, 2006.

44. PUNGLIA, R.S.; D'AMICO, A.V.; CATALONA, W.J. y cols.: "Effect of verification bias on screening for prostate cancer by measurement of pros- 
tate-specific antigen”. N. Engl. J. Med., 349: 335, 2003.

45. ETZIONI, R.; PENSON, D.F.; LEGLER, J.M. y cols.: "Overdiagnosis due to prostate-specific antigen screening: lessons from U.S. prostate cancer incidence trens". J. Natl. Cancer Inst., 94: 981, 2002.

**46. BILL-AXELSON, A.; HOLMBERG, L.; RUUTU, M. y cols.: "Radical prostatectomy versus watchful waiting in early prostate cancer". N. Engl. J. Med., 352: 1977, 2005.

47. ALLEPUZ LOSA, C.A.; GIL SANZ, M.J.; GONZALVO IBARRA, A. y cols.: "Diagnóstico precoz del cancer de próstata en población seleccionada. Utilidad del PSAD, PSAD AD y PSA ajustado a la edad en pacientes con PSA entre 4-10 ngr/ml”. Actas Urol. Esp., 21: 344, 1997.

48. DJAVAN, B.; ZLOTTA, A.; KRATZIK, C. y cols.: "PSA, PSA density, PSA density of transition zone, free/total PSA ratio, and PSA velocity for early detection of prostate cancer in men with serum PSA 2.5 to $4.0 \mathrm{ng} / \mathrm{ml}$ ". Urology, 54: 517, 1999.

*49. CARLSON, G.D.; CALVANESE, C.B.; PARTIN, A.W.: "An algorithm combining age, total prostate-specific antigen (PSA), and percent free PSA to predict prostate cancer: results on 4298 cases". Urology, 52: 455, 1998.

50. BARBAGELATA, A.; VEIGA, F.; BLANCO, A. y cols.: "A model to predict cancer in patients undergoing a first biopsy. Experience over 2683 biopsies". BJU Int., 2: 3, 2004.

51. GÓMEZ VEIGA, F.; PERTEGA, S.; SUÁREZ, G. y cols.: "The usefulness of neural networks to diagnose prostatic cancer". Eur. Urol., 39: 183, 2001.

52. GARZOTTO, M.; BEER, T.M.; HUDSON, R.G. y cols.: "Improved detection of prostate cancer using classificacion and regression tree analysis". J. Clin. Oncol., 23: 4322, 2005.

53. NADLER, R.B.; HUMPHREY, P.A.; SMITH, D.S. y cols.: "Effect of inflammation and bening prostatic hyperplasia on elevated serum prostate specific antigen levels". J. Urol., 154: 407, 1995.

54. LUJAN, M.; PAEZ, A.; SÁNCHEZ, E. y cols.: "Prostate specific antigen variation in patients without clinically evident prostate cancer". J. Urol., 162: 1311, 1999.

*55. BENSON, M.C.; WHANG, I.S.; OLSSON, C.A. y cols.: "The use of prostate specific antigen density to enhance the predictive value of intermediate levels of serum prostate specific antigen”. J. Urol., 147: 817, 1992.

56. BRAWER, M.K.; ARAMBURU, E.A.; CHEN, G.L. y cols.: "The inability of prostate specific antigen index to enhance the predictive value of PSA in the diagnosis of prostatic carcinoma”. J. Urol., 150: 369, 1993.

57. METTLIN, C.; LITTRUP, P.J.; KANE, R.A. y cols.: "Relative sensitivity and specificity of serum prostate specific antigen (PSA) level compared with age-referenced PSA, PSA density and PSA change: Data from the American Cancer Society National Prostate Cancer Detection Project". Cancer, 74: 1615, 1994.

58. SANZ CHINESCA, S.; MARTÍNEZ JABALOYAS, J.M.; BORONAT TORMO, F. y cols.: "Factores que influyen en la variabilidad de la concentración sérica de PSA en pacientes con hiperplasia benigna de próstata". Actas Urol. Esp., 20: 867, 1996.

*59. OESTERLING, J.E.; JACOBSEN, S.J.; CHUTE, C.G. y cols.: "Serum prostate specific antigen in a communitybased population of healthy men: Establishment of age-specific reference ranges". JAMA, 270: 860, 1993.

60. PUNGLIA, R.S.; D’AMICO, A.V.; CATALONA, W.J. y cols.: "Impact of age, benign prostatic hyperplasia, and cancer on prostate-specific antigen level". Cancer, 106: 1507, 2006.

61. MOROTE, J.; LÓPEZ, M.; ENCABO, G. y cols.: "Effect of inflammation and benign prostatic enlargement on total and percent free serum prostatic specific antigen". Eur. Urol., 37: 537, 2000.

62. GÓMEZ PÉREZ, L.; BUDÍA ALBA, A.; REDÓN, B. y cols.: "Influencia de la inflamación crónica prostática en muestras de biopsia sextante en los niveles séricos de PSA total y PSA libre". Actas Urol. Esp., 29: 170, 2005.

63. MARKS, L.S.; ANDRIOLE, G.L.; FITZPATRICK, J.M. y cols.: "The interpretation of serum prostate specific antigen in men receiving 5alpha-reductase inhibitors: a review and clinical recommendations". J. Urol., 176: 868, 2006.

*64. CARTER, H.B.; PEARSON, J.D.; METTER, E.J. y cols.: "Longitudinal evaluation of prostate-specific antigen levels in men with and without prostate disease". JAMA, 267: 2215, 1992.

*65. D'AMICO, A.V.; CHEN, M.H.; ROEHL, K.A. y cols.: "Preoperative PSA velocity and the risk of death from prostate cancer after radical prostatectomy”. N. Engl. J. Med., 351: 125, 2004.

66. RAAIJMAKERS, R.; WILDHAGEN, M.F.; ITO, K. y cols.: "Prostate-specific antigen change in the european randomized study of screening for prostate cancer, section Rotterdam". Urology, 63: 316, 2004.

67. PRESTIGIACOMO, A.F.; STAMEY, T.A.: "Physiological variation of serum prostate specific antigen in the 4.0 to $10.0 \mathrm{ng} . / \mathrm{ml}$. range in male volunteers". J. Urol., 155: 1977, 1996.

68. BODDY, J.L.; PIKE, D.J.; AL-HAYEK, S. y cols.: "An Elevated PSA, which normalizes, does not exclude the presence of prostate cancer". Prostate Cancer Prostatic Dis., 8: 349, 2005.

*69. EASTHAM, J.A.; RIEDEL, W.; SCARDINO, P.T. y cols.: "Begg CB for the Polyp Prevention Trial Study Group. Variation of serum prostate-specific 
antigen levels: an evaluation of year-to- year fluctuations". JAMA, 289: 2695, 2003.

70. SEMJONOW, A.G.; OBERPENNING, F.H.; SCHMID, B.P. y cols.: "The clinical impact of different assays for prostate specific antigen". BJU Int., 86: 590, 2000.

71. STEPHAN, C.; KLAAS, M.; MÜLLER, C. y cols.: "Interchangeability of measurements of total and free prostate-specific antigen in serum with 5 frequently used assay combinations: an update". Clin. Chem., 52: 59, 2006.

72. STAMEY, T.A.; CALDWELL, M.; McNEAL, J.E. y cols.: "The protate specific antigen era in the united states is over for prostate cancer: what happened in the last 20 years?". J. Urol., 172: 1297, 2004.

*73. THOMPSON, I.M.; ANKERST, D.P.; CHI, C. y cols.: "Operating characteristics of prostate-specific antigen in men with an initial PSA level of 3.0 ng/mL or lower". JAMA, 294: 66, 2005.

74. FREEDLAND, S.J.; MANGOLD, L.A.; WALSH, P.C. y cols.: "The prostatic specific antigen era is alive and well: prostatic specific antigen and biochemical progression following radical prostatectomy". J. Urol., 174: 1276, 2006.

75. GONZALGO, M.L.; ISAACS, W.B.: "Molecular pathways to prostate cancer". J. Urol., 170: 2444, 2003.

76. TROYER, D.A.; MUBIRU, J.; LEACH, R.J. y cols.: "Promise and challenge: markers of prostate cancer detection, diagnosis and prognosis". Dis. Markers, 20: 117, 2004.

**77. WANG, X.; YU, J.; SREEKUMAR, A. y cols.: "Autoantibody signatures in prostate cancer". N. Engl. J. Med., 353: 1224, 2005.

*78. RUBIN, M.A.; ZHOU, M.; DHANASEKARAN, S.M. y cols.: “_-Methylacyl coenzyme. A racemase as a tissue biomarker for prostate cancer". JAMA, 287: 1662, 2002.

**79. SREEKUMAR, A.; LAXMAN, B.; RHODES, D.R. y cols.: "Humoral immune response to alphamethylacyl-Co. A racemase and prostate cancer". J. Natl. Cancer Inst., 96: 834, 2004.

*80. TRICOLI, J.V.; SCHOENFELDT, M.; CONLEY, B.A.: "Detection of prostate cancer and predicting progression: current and future diagnostic markers”. Clin. Cancer Res., 10: 3943, 2004.

81. GOESSI, C.; MULLER, M.; HEICAPPELL, R. y cols.: "DNA-based detection of prostate cancer in urine after prostatic massage". Urology, 58: 335, 2001.

82. BOTCHKINA, G.I.; KIM, R.H.; BOTCHKINA, I.L. y cols.: "Noninvasive detection of prostate cancer by quantitative analysis of telomerase activity". Clin. Cancer Res., 11: 3243, 2005.

83. DASI, F.; MARTINEZ-RODES, P.; MARCH, J.A. y cols.: "Real-time quantification of human telomerase reverse transcriptase mRNA in the plasma of patients with prostate cancer". Ann. NY Acad. Sci., 1075: 204, 2006.

84. OSMAN, I.; MIKHAIL, M.; SHUCH, B. y cols.: "Serum levels of shed HER2/neu protein in men with prostate cancer correlate with disease progression”. J. Urol., 174: 2174, 2005.

85. OREGAWA, T.; KINJO, M.; NUTAHARA, K. y cols.: "Pretreatment serum level of HER2/nue as a prognostic factor in metastatic prostate cancer patients about to undergo endocrine therapy". Int. J. Urol., 13: 1197, 2006.

86. GILS, M.P.M.Q.; STENMAN, U.H.; SCHALKEN, J.A. y cols.: "Innovations in serum and urine markers in prostate cancer current European research in the P-Mark project". Eur. Urol., 48: 1031, 2005.

87. ORNSTEIN, D.K.; RAYFORD, W.; FUSARO, V.A. y cols.: "Serum proteomic profiling can discriminate prostate cancer from benign prostates in men with total prostate specific antigen levels between 2.5 and $15.0 \mathrm{ng} / \mathrm{ml}$ ". J. Urol., 174: 1302, 2004.

88. BANEZ, L.L.; PRASANNA, P.; SUN, L. y cols.: "Diagnostic potential of serum proteomic patterns in prostate cancer". J. Urol., 170: 442, 2003.

89. LI, J.; WHITE, N.; ZHANG, Z. y cols.: "Detection of prostate cancer using serum proteomics pattern in a histologically confirmed population". J. Urol., 171: 1782, 2004.

90. HERNÁNDEZ, J.; CANBY-HAGINO, E.; THOMPSON, I.M.: "Biomarkers for the detection and prognosis of prostate cancer". Curr. Urol. Rep., 6: 171, 2005.

91. YOUNG, C.Y.; ANDREWS, P.E.; MONTGOMERY, B.T. y cols.: "Tissue-specific and hormonal regulation of human prostate-specific glandular kallikrein". Biochemistry, 31: 818, 1992.

92. DIAMANDIS, E.P.; YOSUEF, G.M.: "Human tissue kallikreins: a family of new cancer biomarkers". Clin. Chem., 48: 1198, 2002.

93. STEUBER, T.; NIEMELA, P.; HAESE, A. y cols.: "Association of free-prostate specific antigen subfraction and human glandular kallikrein 2 with volume of bening and malignant prostatic tissue". Prostate, 63: 13, 2005.

*94. MIKOLAJCZYK, S.D.; MARKER, K.M.; MILLAR, L.S. y cols.: "A truncated form of prostatespecific antigen is a more specific serum marker of prostate cancer". Cancer Res., 61: 6958, 2001.

95. LINTON, H.J.; MARKS, L.S.; MILLAR, L.S. y cols.: "Benign prostatespecific antigen (BPSA) in serum is increased in benign prostate disease". Clin. Chem., 49: 253, 2003.

96. CATALONA, W.J.; BARTSCH, G.; RITTENHOUSE, H.G. y cols.: "Serum pro prostate specific antigen improves cancer detection compared to free and complexed prostate specific antigen in men with prostate specific antigen 2 to $4 \mathrm{ng} / \mathrm{ml}$ ". J. Urol., 170: 2181, 2003. 